\title{
Cultivating a Three-dimensional Reconstructed Human Epidermis at a Large Scale
}

\author{
Irini M. Dijkhoff ${ }^{1}$, Benedetta Petracca ${ }^{1,2}$, Roxane Prieux ${ }^{3}$, Giuseppe Valacchi ${ }^{3,4}$, Barbara Rothen-Rutishauser ${ }^{1}$, Marc Eeman $^{2}$ \\ ${ }^{1}$ Adolphe Merkle Institute, University of Fribourg ${ }^{2}$ Dow Silicones Belgium SRL ${ }^{3}$ Department of Biomedical and Specialist Surgical Sciences, University of \\ Ferrara ${ }^{4}$ Department of Animal Sciences, Plants for Human Health Institute, North Carolina State University
}

\section{Corresponding Author}

Marc Eeman

marc.eeman@dow.com

\section{Citation}

Dijkhoff, I.M., Petracca, B., Prieux, R., Valacchi, G., Rothen-Rutishauser, B., Eeman, M. Cultivating a Threedimensional Reconstructed Human Epidermis at a Large Scale. J. Vis. Exp. (171), e61802, doi:10.3791/61802 (2021).

\section{Date Published}

May 28, 2021

DOI

$10.3791 / 61802$

URL

jove.com/video/61802

\section{Abstract}

A three-dimensional human epidermis model reconstructed from neonatal primary keratinocytes is presented. Herein, a protocol for the cultivation process and the characterization of the model is described. Neonatal primary keratinocytes are grown submerged on permeable polycarbonate inserts and lifted to the air-liquid interface three days after seeding. After fourteen days of stimulation with defined growth factors and ascorbic acid in high calcium culture medium, the model is fully differentiated. Histological analysis revealed a completely stratified epidermis, mimicking the morphology of native human skin. To characterize the model and its barrier functions, protein levels and localization specific for early-stage keratinocyte differentiation (i.e., keratin 10), late-stage differentiation (i.e., involucrin, loricrin, and filaggrin) and tissue adhesion (i.e., desmoglein 1), were assessed by immunofluorescence. The tissue barrier integrity was further evaluated by measuring transepithelial electrical resistance. Reconstructed human epidermis was responsive to proinflammatory stimuli (i.e., lipopolysaccharide and tumor necrosis factor alpha), leading to increased cytokine release (i.e., interleukin 1 alpha and interleukin 8). This protocol represents a straightforward and reproducible in vitro method to cultivate reconstructed human epidermis as a tool to assess environmental effects and a broad range of skin-related studies.

\section{Introduction}

The epidermis is the outermost layer of the skin, at the direct interface between the human body and the external environment. Its main functions are to provide protection and hydration ${ }^{1}$. The epidermis acts as an effective physical barrier against external agents and prevents excessive water loss from the body. These skin functions mainly depend on the cellular arrangement in the outermost layers of the skin, the composition, and organization of intercellular lipids ${ }^{2}$. The epidermis is primarily composed of keratinocytes that migrate upwards to the outer side of the tissue and 
undergo differentiation. There are 4-5 epidermal layers that are characterized by their stage of differentiation. From the inside to the outside, the epidermal layers start from the viable epidermis, i.e., the stratum basale (SB), the stratum spinosum (SS), and the stratum granulosum (SG), to the non-viable uppermost layer, i.e., the stratum corneum $(\mathrm{SC})^{3}$. The basal layer is mainly composed of proliferating keratin-enriched keratinocytes, which migrate through the SS upon differentiation ${ }^{4}$. During keratinocyte maturation, various changes in protein expression and structure occur. Keratinocytes adhere through the formation of desmosomal junctions ${ }^{5}$. In the SG, the generation of lamellar bodies is initiated. They consist of lipid precursors and enzymes that are crucial for the formation of the skin barrier function ${ }^{6}$. The SG is also characterized by the presence of keratohyalin granules in the cytoplasm of the keratinocytes. At the interface with the SC, the content of the lamellar bodies is extruded into the intercellular spaces and the non-polar lipids such as ceramides, cholesterol, and free fatty acids organize into stacked lamellar lipid bilayers to form the extracellular lipid matrix $^{7}$. In the SC, cells lose all cellular organelles including the nucleus, due to enzymatic degradation processes and adopt a flattened morphology. They are surrounded by a cornified envelope made of cross-linked protein layers, and are referred to as corneocytes ${ }^{8}, 9$. Desmosomal components are cross-linked to the cornified envelope to form corneodesmosomes and bind the corneocytes together. The resulting epithelium is continually renewed from stem cells, with a turnover time of approximately $5-6$ weeks $^{10}$. The differentiation process of the keratinocytes, which results in a fully stratified epidermis, is crucial for the formation of the barrier function of the skin ${ }^{11}$.

During wounding and inflammation, keratinocytes induce changes in adhesion molecules and surface receptors and trigger proinflammatory responses via secretion of cytokines, chemokines, and antimicrobial peptides ${ }^{12}$. The skin is not only a physical barrier against exogenous substances; it also acts as an immune sensor upon exposure to pathogens. In addition, it regulates the diffusion of several substances across its layers, such as water content to protect the human body from dehydration. The skin is also involved in the synthesis of vitamin $D$ and has various other metabolic functions ${ }^{3}, 13,14$.

To assess the adverse effects of exogenous substances, toxicologists have relied for decades on animal testing, but nowadays it is not the preferred approach. Besides having limited predictive capacity for human toxicity, animal models involve numerous ethical issues. The ban on animal testing in the cosmetic industry and the recommendation to follow the 3R principle (i.e., Replacement, Reduction, and Refinement) in research have led to the development of alternative test methods based on in vitro approaches ${ }^{15}$.The first in vitro skin cell models have already been described in the 90's, and an impressive development from simple human keratinocyte mono-cultures to fully differentiated epidermis and full-thickness models has been achieved ${ }^{16}$. Nowadays, skin tissue engineering has gained importance in both the pharmaceutical and dermato-cosmetic fields. In the last two decades, several companies have commercialized threedimensional (3D) reconstructed human epidermis (RhE) that represent standardized and reproducible tools for skin-related studies. Several commercial RhE models are accepted for in vitro skin testing of chemicals according to OECD guidelines for the testing of skin irritation ${ }^{17,} 18$ (i.e., test guideline $439^{19}$ ) and skin corrosion ${ }^{20}$ (i.e., test guideline $\left.431^{21}\right)$. The in vitro test for skin sensitization ${ }^{22}$ (i.e., SENS-IS assay) is currently in the approval track and under peer-review ${ }^{23}$. There are also numerous other 
assays developed that utilize commercial RhE models, to evaluate phototoxicity ${ }^{24}$, to test drug formulations ${ }^{25}$, cosmetic formulations and active ingredients ${ }^{26}$, to study the skin barrier function ${ }^{27}$ and to test the biological response to environmental stressors $28,29,30,31$.

In addition to commercially available 3D skin models, multiple research groups have developed their own

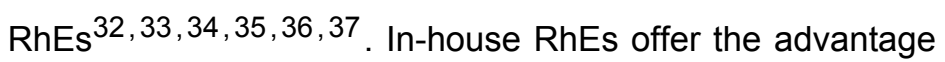
for controlling the culture conditions according to the purpose of the study. Specifically, researchers can select the type and the source of the keratinocytes to be used for the reconstitution of their 3D epidermal model (i.e., primary vs. immortalized, neonatal vs. aged, single vs. pooled random donors, sex, ethnicity, individual living habits such as smoking, etc.). They have the possibility to vary the composition of the culture medium and incorporate growth factors, vitamins, or other compounds that can modulate the expression of target proteins or lipids. With in-house RhEs, researchers can also investigate biological responses and biomechanical properties as a function of the differentiation state of the 3D model. In addition to those intuitive parameters, there are continuous efforts to increase the complexity of 3D skin models and make them more physiologically relevant, for instance by adding other epidermal cell types (e.g. melanocytes and immune cells) ${ }^{38,} 39$, by culturing the keratinocytes on top of a fibroblast-populated collagen matrix $40,41,42$, and by including components of the vascular network $43,44,45$.

Although it is possible to tune the culture conditions according to specific needs, there are parameters that must be respected to guarantee both the quality and relevance of a RhE. To cultivate RhE tissues, normal human epidermal keratinocytes (NHEKs) are seeded into specific permeable culture inserts whose porous synthetic membrane separates the wells into two compartments, i.e., the apical and basolateral compartment. The porosity of the membrane (i.e., a pore size of $0.4 \mu \mathrm{m}$ ) is such that it allows the formation of a cell monolayer in the apical compartment with no migration of cells to the basal insert side, and the feeding of the keratinocytes with essential nutrients from the culture medium contained in the basolateral compartment. At the beginning of the reconstitution process, NHEKs are cultured in submerged conditions for a few days to allow their adhesion onto the membrane. The calcium level in both compartments is increased compared to the calcium concentration used for the 2D culture of NHEKs to slow down the proliferation of cells and promote instead their differentiation ${ }^{46}$. An epidermal calcium gradient is essential to regulate the barrier formation and homeostasis 47,48 . High calcium levels (i.e., up to $1.5 \mathrm{mM}$ ) promote the formation of intercellular junctions and modulate the formation of the cornified envelope during terminal differentiation ${ }^{49}$. Once keratinocytes form a continuous and tightly adherent monolayer on the supporting membrane, the medium from the apical compartment is removed and the culture process continues at the airliquid interface $(A L I)$ to stimulate stratification and establish an epidermal barrier 50,51 . Specific culture conditions are crucial to obtain a fully stratified epithelium ${ }^{36}$. During the reconstitution process at $\mathrm{ALI}$, the medium in the basolateral compartment is supplemented with keratinocyte growth factor (KGF), insulin, calcium, and ascorbic acid. Ascorbic acid plays a major role in the formation of an appropriate SC lipid barrier, closely resembling that of the native human skin $^{52}$. Keratinocytes grown in ascorbic acid-supplemented medium demonstrate a differentiated phenotype, with an enhanced number of keratohyalin granules, as well as organized intercellular lipid lamellae in the interstices of the corneocytes $^{52}$. Such supplementation is essential to improve 
epidermal barrier function by increasing cornified envelope content and avoiding depletion of hydrophilic antioxidant stores $^{53}, 54$. KGF, an important paracrine mediator of epidermal proliferation and differentiation, is used to stimulate the NHEKs ${ }^{55}$.

The main downsides of in-house RhEs include the loss of standardization between research institutions and increased labor intensity and time consumption (up to 3 weeks compared to the ready-to-use commercial models). The aim of the present paper is to address these drawbacks, setting the basis for production at a larger scale. In addition to the abovementioned advantages of in-house RhEs, the current protocol aims to reduce the intra- and inter-variability among tissues, to reduce contamination risks, and to streamline the cultivation process.

The current protocol describes a reproducible and robust method to cultivate RhEs using neonatal NHEKs. Moreover, it shows representative results of the characterization of the RhEs morphology, barrier integrity, and expression of proteins that are specific for epidermal differentiation. RhEs morphological structure was examined using hematoxylin and eosin (H\&E) staining and transmission electron microscopy (TEM). To evaluate the barrier integrity, the transepidermal electrical resistance (TEER) and the exposure time to Triton $\mathrm{X}-100$ to reduce $50 \%$ of the tissue viability (ET50) were measured. The formation of desmosomal junctions (i.e., desmoglein 1) was analyzed by immunofluorescence (IF) to evaluate keratinocyte adhesion. The formation of epidermal structural proteins (i.e., involucrin, loricrin, and filaggrin) was evaluated and detected with IF. These proteins are involved in the formation of the highly cross-linked protein envelope that surrounds SC corneocytes and as a result are important markers for late-stage epidermal differentiation ${ }^{56}$, 57. Additionally, IF was used to analyze keratin 10 , a protein induced in early-stage differentiated cells in the $S^{58}$ and found inside all differentiated layers. Finally, the RhE's response to proinflammatory stimuli (i.e., lipopolysaccharide and tumor necrosis factor alpha) was investigated. The levels of interleukin 1 alpha (IL-1 $\alpha$ ) and interleukin 8 (IL-8) were measured in the cell culture media, using enzyme-linked immunosorbent assays (ELISA).

\section{Protocol}

Review and adhere to national and international ethical considerations and conditions related to the use of human tissues or cells before planning and executing any research activity involving this protocol.

NOTE: All steps of this protocol must be carried out in aseptic conditions. Biosafety Level 2 practices are the minimum requirement for the cultivation of RhEs. All necessary safety precautions must be taken when handling the chemicals/ reagents described in this protocol.

\section{Preparation of cell culture media}

NOTE: There are three different types of serum-free culture media used for the cultivation of RhEs (Table 1): (i) the basal medium with low calcium level $\left(60 \mu \mathrm{M} \mathrm{Ca}^{2+}\right)$ used for the 2D culture of NHEKs; (ii) the submerged medium with high calcium level $\left(1.5 \mathrm{mM} \mathrm{Ca}^{2+}\right)$ used for the seeding of NHEKs into the cell culture insert system; and (iii) the air-liquid interface $(A L I)$ medium with high calcium level $\left(1.5 \mathrm{mM} \mathrm{Ca}^{2+}\right)$, ascorbic acid, and keratinocyte growth factor (KGF).

\begin{tabular}{|c|c|c|}
\hline Medium & Medium information & Required quantity \\
\hline \multirow{2}{*}{ Basal medium } & Keratinocyte growth medium & $36 \mathrm{~mL} / 24$ wells
\end{tabular}




\begin{tabular}{|c|c|c|}
\hline & $+1 \%[v / v]$ HKGS & \\
\hline & $+1 \%[v / v] 100 x$ antibiotic-antimycotic & \\
\hline \multirow[t]{4}{*}{ Submerged medium } & Keratinocyte growth medium & \multirow[t]{4}{*}{$36 \mathrm{~mL} / 24$ wells } \\
\hline & $+1 \%[v / v]$ HKGS & \\
\hline & $+1 \%[v / v] 100 x$ antibiotic-antimycotic & \\
\hline & $+1.5 \mathrm{mM} \mathrm{Ca}^{2+}$ & \\
\hline \multirow[t]{6}{*}{ Air-liquid interface medium } & Keratinocyte growth medium & \multirow[t]{6}{*}{$216 \mathrm{~mL} / 24$ wells } \\
\hline & $+1 \%[v / v]$ HKGS & \\
\hline & $+1 \%[v / v] 100 x$ antibiotic-antimycotic & \\
\hline & $+1.5 \mathrm{mM} \mathrm{Ca}^{2+}$ & \\
\hline & $+50 \mu \mathrm{g} / \mathrm{mL}$ ascorbic acid & \\
\hline & $+10 \mathrm{ng} / \mathrm{mL}$ keratinocyte growth factor & \\
\hline
\end{tabular}

Table 1. Summary table of the different culture media used to cultivate RhEs. List of different culture media with supplements.

1. Prepare the basal medium.

1. Supplement the bottle of $500 \mathrm{~mL}$ of keratinocyte growth medium (Table of Materials) with $5 \mathrm{~mL}$ of human keratinocyte growth supplements (HKGS) in order to reach final concentrations of $0.2 \%[\mathrm{v} /$ v] bovine pituitary extract (BPE), $0.2 \mathrm{ng} / \mathrm{mL}$ human recombinant epidermal growth factor (EGF), 0.18 $\mu \mathrm{g} / \mathrm{mL}$ hydrocortisone, $5 \mu \mathrm{g} / \mathrm{mL}$ bovine transferrin, and $0.01 \mu \mathrm{g} / \mathrm{mL}$ of recombinant human insulin-like growth factor-I (IGF-I).

2. Add $5 \mathrm{~mL}$ of $100 \mathrm{x}$ antibiotic-antimycotic solution containing 10,000 units $/ \mathrm{mL}$ of penicillin, $10,000 \mu \mathrm{g} /$ $\mathrm{mL}$ of streptomycin, and $25 \mu \mathrm{g} / \mathrm{mL}$ of amphotericin B.

2. Prepare the submerged medium.
1. Supplement the bottle of $500 \mathrm{~mL}$ of keratinocyte growth medium (Table of Materials) with $5 \mathrm{~mL}$ of HKGS in order to reach final concentrations of $0.2 \%$ [v/v] BPE, $0.2 \mathrm{ng} / \mathrm{mL}$ human recombinant EGF, 0.18 $\mu \mathrm{g} / \mathrm{mL}$ hydrocortisone, $5 \mu \mathrm{g} / \mathrm{mL}$ bovine transferrin, and $0.01 \mu \mathrm{g} / \mathrm{mL}$ of human recombinant IGF-I.

2. Add $5 \mathrm{~mL}$ of $100 x$ antibiotic-antimycotic solution.

3. Add $5 \mathrm{~mL}$ of a $0.144 \quad \mathrm{M} \mathrm{CaCl} 2$ (calcium chloride) stock solution to reach a final concentration of $1.5 \mathrm{mM} \mathrm{Ca}^{2+}$.

NOTE: The calcium concentration is already increased during the submerged phase to stimulate the differentiation of the keratinocytes and initiate the stratification process ${ }^{49}$. 
3. Prepare the air-liquid interface (ALI) medium.

1. Supplement one bottle of $500 \mathrm{~mL}$ of keratinocyte growth medium (Table of Materials) with $5 \mathrm{~mL}$ of HKGS in order to reach final concentrations of $0.2 \%$ [v/v] BPE, $0.2 \mathrm{ng} / \mathrm{mL}$ human recombinant EGF, 0.18 $\mu \mathrm{g} / \mathrm{mL}$ hydrocortisone, $5 \mu \mathrm{g} / \mathrm{mL}$ bovine transferrin, and $0.01 \mu \mathrm{g} / \mathrm{mL}$ of human recombinant IGF-I.

2. Add $5 \mathrm{~mL}$ of $100 \mathrm{x}$ antibiotic-antimycotic solution.

3. Add $5 \mathrm{~mL}$ of a $0.144 \mathrm{M} \mathrm{CaCl}_{2}$ stock solution to reach a final concentration of $1.5 \mathrm{mM} \mathrm{Ca}^{2+}$.

4. Add $1 \mathrm{~mL}$ of a $25 \mathrm{mg} / \mathrm{mL}$ ascorbic acid stock solution to reach a final concentration of $50 \mu \mathrm{g} / \mathrm{mL}$ ascorbic acid.

5. Add $50 \mu \mathrm{L}$ of a $100 \mu \mathrm{g} / \mathrm{mL} \mathrm{KGF}$ in $1 \%$ [w/v] bovine serum albumin in phosphate-buffered saline (PBS) stock solution to reach a final concentration of $10 \mathrm{ng} /$ $\mathrm{mL}$ KGF

NOTE: Since ascorbic acid is sensitive to oxidation, it is recommended to use a stable ascorbic acid-derivative, such as magnesium I-ascorbyl-2phosphate ${ }^{59}$ or L-ascorbic acid 2-phosphate sesquimagnesium ${ }^{60}$. If ascorbic acid is used, it is recommended to freshly supplement the ALI medium with ascorbic acid before each refresh.

\section{Culture of NHEKs}

NOTE: Since primary human keratinocytes remain proliferative upon their fourth or fifth passage ${ }^{61}$, NHEKs in their third passage are used for the cultivation of RhEs. Primary keratinocytes should be handled very carefully due to their high sensitivity. Careful and slow pipetting of cell suspensions at any time is very important, to not disturb the condition of the cells.

1. Thaw a vial with $1 \times 10^{6}$ cryopreserved NHEKs in a water bath at $37^{\circ} \mathrm{C}$, by submerging part of the vial in the water. Incubate the vial for 1-2 minutes in the water bath, until only a small sliver of ice is visible.

CAUTION: Do not submerge the whole vial in the water bath to avoid contaminations. Do not thaw the cells longer than 2 minutes; this can reduce the cell viability. Wipe the vial with a $70 \%$ ethanol solution before transferring the tube into the laminar hood.

2. Resuspend the cells very carefully, by pipetting up and down 2-3 times. Transfer the cell suspension into two T75 flasks containing a total of $15 \mathrm{~mL}$ of pre-warmed thawing medium, resulting in a seeding density of $6.7 \times 10^{4}$ cells/ $\mathrm{cm}^{2}$.

NOTE: For the first two passages and the thawing of cryopreserved NHEKs, cell culture medium is used according to the supplier's recommendations.

3. Place the flasks into the cell culture incubator $\left(37^{\circ} \mathrm{C}, 5 \%\right.$ $\mathrm{CO}_{2}$, and $95 \%$ relative humidity $\left.(\mathrm{RH})\right)$.

4. After approximately 24 hours, replace the thawing medium by the basal medium to remove dimethyl sulfoxide (DMSO) from the keratinocyte freezing solution.

5. Refresh the basal medium every two days.

6. After 4-6 days of cultivation, the cells should be around $80 \%$ confluent and ready for seeding in inserts for the cultivation of RhEs.

NOTE: Keratinocytes should be grown to maximum $80 \%$ confluence to preserve their proliferative capacity ${ }^{62}$. The 
number of cells to be thawed must take into consideration several parameters, such as the cell passage number, the cell viability upon thawing, the seeding efficiency as well as the doubling time.

\section{Seeding of NHEKs}

NOTE: This protocol is designed for use within a 24-well carrier plate format. If other plate formats are required (e.g., 12-well or 6-well format), optimizations in the seeding density and medium volume should be considered. Figure 1 summarizes a proposed timeline for RhE cultivation and shows the cultivation conditions. 
1. seed $1.8 \times 10^{5}$ cells in $500 \mu \mathrm{L} /$ insert

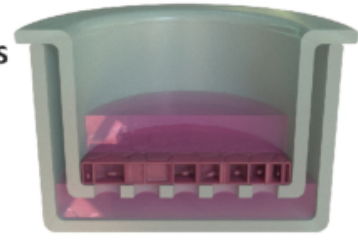

Day -3

$\downarrow$

2. remove medium

from apical compartment

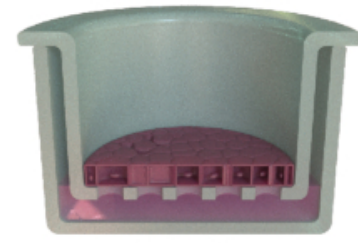

air-liquid interface medium keratinocyte growth medium $+1.5 \mathrm{mM} \mathrm{Ca}^{2+}$

$+50 \mu \mathrm{g} / \mathrm{mL}$ ascorbic acid

$+10 \mathrm{ng} / \mathrm{mL}$ KGF

Day 0

3. refresh medium every 2-3 days to stimulate differentiation

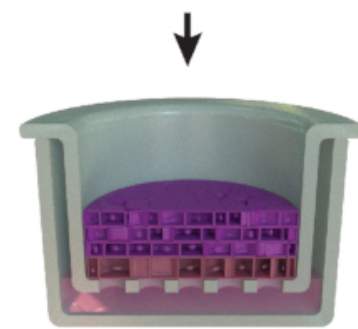

Day 2/Day 4/Day7/Day 9/Day 11

4. exposure of reconstructed human epidermis

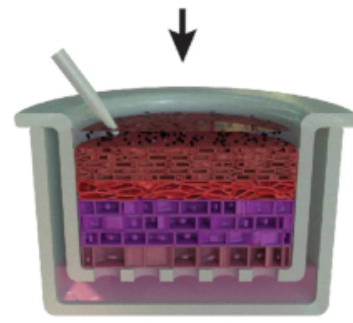

submerged medium

Day 14

$\downarrow$

5. harvest

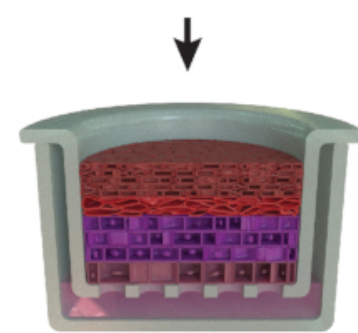

keratinocyte growth medium $+1.5 \mathrm{mM} \mathrm{Ca}^{2+}$

Day 15/Day 16 
Figure 1: Schematic timeline of the reconstitution protocol. Presentation of the RhE model preparation, cultivation process, and application (exposure to chemical substance). The scheme includes the appropriate cell culture media types for each step. Please click here to view a larger version of this figure.

1. Pre-fill 24-well plates with $1.5 \mathrm{~mL}$ of submerged medium, ideally using a dispenser pipette.

2. Remove the basal medium from the T75 flasks used for the culture of the NHEKs.

3. Rinse the cells by adding $5 \mathrm{~mL}$ of pre-warmed PBS to each T75 flask.

4. Remove PBS from the flasks.

NOTE: This step is crucial, since the medium contains proteins and calcium that will inhibit the trypsin activity.

5. Add $2-3 \mathrm{~mL}$ of pre-warmed $0.05 \%[\mathrm{v} / \mathrm{v}]$ trypsin/ethylene diamine tetra acetic acid (EDTA) to each T75 flask. Make sure that the trypsin solution is equally distributed on the cell culture area of the flask.

CAUTION: The $2 \mathrm{~mL}$ volume is based on the $80 \%$ confluence mentioned above. Use $3 \mathrm{~mL}$ for flasks with a higher confluency.

6. Place the flasks for 4 minutes in the cell culture incubator $\left(37^{\circ} \mathrm{C}, 5 \% \mathrm{CO}_{2}\right.$, and $\left.95 \% \mathrm{RH}\right)$. Check whether the cells detach using the microscope at a 10x magnification. Rap the flask against the palm of the hand to help the cells release from the surface of the flask. Detached cells can be observed as rounded cells floating in the trypsin solution.

CAUTION: Do not incubate the cells in trypsin for longer than 6 minutes. Over-trypsinization can damage the cells and decrease their adherence ${ }^{63}$.
7. Once all the cells are detached, add an equal volume (i.e., 2-3 mL) of pre-warmed trypsin inhibitor to each T75 flask.

8. Transfer the cell suspension from the flasks to a centrifuge tube.

9. Rinse the flasks with $5 \mathrm{~mL}$ of pre-warmed PBS and transfer it to the centrifuge tube containing the cell suspension.

NOTE: Make sure that most of the cells are collected by checking the number of residual cells in the flasks under the microscope. The surface of the flask should be $95 \%$ empty. If this is not the case it is possible to repeat the trypsinization step (3.3-3.9). Note however that retrypsinization should be avoided.

10. Centrifuge the harvested cells at $400 \times g$ for $5 \mathrm{~min}$.

11. Carefully discard most of the supernatant, leaving approximately $100-200 \mu \mathrm{L}$ in the tube.

CAUTION: Do not aspirate the pellet during this procedure.

12. Gently resuspend the pellet of cells in a low volume of submerged medium, pipette up and down 5-10 times to ensure a uniform cell suspension. Start with a low volume (i.e., $500 \mu \mathrm{L}$ ) to avoid the formation of cell aggregates and add up to $1 \mathrm{~mL}$ of submerged medium in total per initial T75 flask.

NOTE: Gently flick the tube with fingers to carefully dissolve a part of the cell pellet in the supernatant.

13. Count the cells in the suspension using the trypan blue exclusion method. 
1. Dilute $0.4 \%[\mathrm{v} / \mathrm{v}]$ trypan blue stain and the cell suspension in a $1: 1$ ratio, by adding $10 \mu \mathrm{L}$ of $0.4 \%$ [v/ v] trypan blue stain to $10 \mu \mathrm{L}$ of cell suspension. Add $10 \mu \mathrm{L}$ of the solution to a counting slide. Measure the cell count immediately after mixing the cell suspension with trypan blue, since trypan blue starts to decrease cell viability after exposure longer than $1 \min ^{65}$.

CAUTION: Trypan blue was shown to be a potential mutagen, carcinogen, and teratogen ${ }^{64}$. Handle the dye with care and dispose of the waste safely according to local laboratory regulations.

NOTE: An alternative approach to the use of trypan blue is the non-hazardous dye Erythrosin $\mathrm{B}^{66}$.

14. Dilute the cell suspension with additional submerged medium to reach a concentration of $3.525 \times 10^{5}$ cells/ $\mathrm{mL}$ in submerged medium by adding the volume $\mathrm{V}_{2}$ as shown in equation 1:

$V_{2}=C_{1} * \frac{V_{1}}{C_{2}}-V_{1}$

$\mathrm{C}_{1}=$ counted cell concentration in the cell suspension obtained in 3.12 (cells/mL)

$V_{1}=$ volume used to resuspend the pellet of cells in 3.12 $(\mathrm{mL})$

$\mathrm{C}_{2}=$ targeted cell concentration in the suspension (i.e., $3.525 \times 10^{5}$ cells $/ \mathrm{mL}$ )

$\mathrm{V}_{2}=$ volume to be added to reach the targeted cell concentration $(\mathrm{mL})$

NOTE: The surface area of the recommended culture insert is $0.47 \mathrm{~cm}^{2}$; therefore, the corresponding seeding density is $3.75 \times 10^{5}$ cells $/ \mathrm{cm}^{2}$.
15. Perform a second cell count $\left(\mathrm{C}_{3}\right)$ of the diluted solution obtained in step 3.14. Use equation 2 to calculate the cell suspension volume $\left(V_{4}\right)$ to be seeded into the culture insert:

$V_{4}=C_{3} * \frac{V_{3}}{C_{4}}$

$\mathrm{C}_{3}=$ targeted cell concentration in the suspension (i.e., $3.525 \times 10^{5}$ cells $/ \mathrm{mL}$ )

$V_{3}=$ targeted volume of the cell suspension to be seeded in the culture insert (i.e., $0.5 \mathrm{~mL}$ )

$\mathrm{C}_{4}=$ counted cell concentration in the diluted suspension obtained in 3.14 (cells/mL)

$V_{4}=$ actual volume of the cell suspension to be seeded in the culture insert (mL)

16. Hang the 24 cell culture inserts in the highest position of the recommended carrier plate and transfer the carrier plate to the 24-well plate pre-filled with submerged medium (cf. 3.1).

CAUTION: When transferring the carrier plate to the multi-well plate, ensure that no air bubbles are trapped between the insert membrane and the submerged medium from the basal compartment, as this will affect the feeding of the cells and ultimately compromise the RhE viability and morphology.

17. Add the determined volume $V_{4}$ (from equation 2 ) of the cell suspension to each insert.

NOTE: It is recommended to use the reverse pipetting technique to accurately dispense the cell suspension to the culture inserts.

CAUTION: Make sure not to damage the membrane when dispensing the cell suspension into the culture insert. A precaution is to dispense the cell suspension 
along the wall of the insert system without touching the surface of the membrane.

18. After seeding, incubate the 24 -well plates for $10-15 \mathrm{~min}$ at room temperature, to overcome an edge effect (i.e., nonuniform temperature distribution between all wells ${ }^{67}$ ). Do not move the plates during this time.

19. Transfer the plates to the cell culture incubator $\left(37^{\circ} \mathrm{C}\right.$, $5 \% \mathrm{CO}_{2}$, and $\left.95 \% \mathrm{RH}\right)$. The cells are maintained in submerged conditions for three days.

NOTE: To avoid tissue variability, do not stack the plates in the incubator after seeding to make sure that each insert receives the same amount of heat. After three days (i.e., during ALI cultivation), stacking of plates is possible.

\section{Cultivation at Air-Liquid Interface}

1. After a three-day incubation in the cell culture incubator $\left(37{ }^{\circ} \mathrm{C}, 5 \% \mathrm{CO}_{2}\right.$, and $\left.95 \% \mathrm{RH}\right)$, expose the cells that have adhered to the membrane surface to the ALI by removing the submerged medium from the apical compartment preferably using an aspiration system and a glass Pasteur pipette.

NOTE: Alternatively, the submerged medium from the apical compartment can be removed with a manual micropipette.

2. Fill new 24-well plates with $1.5 \mathrm{~mL}$ of fresh pre-warmed ALI medium and transfer the carrier plates with the culture inserts to the new multi-well plates.

3. Transfer the multi-well plates back to the cell culture incubator $\left(37^{\circ} \mathrm{C}, 5 \% \mathrm{CO}_{2}\right.$, and $\left.95 \% \mathrm{RH}\right)$.

4. Refresh the ALI medium every 2-3 days for 14 days.
5. Perform the refresh in two steps: 1) prepare a new plate containing $1.5 \mathrm{~mL} /$ well of fresh pre-warmed ALI medium and 2) transfer the carrier plate to the new plate.

CAUTION: During the entire reconstitution procedure it is best not to remove the lid covering the carrier plate to keep the RhEs protected from potential contamination.

NOTE: The ALI step is crucial for the development of a stratified epidermal model as it allows terminal differentiation of the keratinocytes ${ }^{68}$. After going to ALI, a visual control of the inserts is required, to check whether there are 'leaky tissues': medium droplets on the tissue surface coming from the basolateral compartment. If the leakage happens at ALI day 3 , gently remove the medium from the culture insert without touching the surface of the tissue. If the leakage persists, it is recommended to discard leaking tissues as it is an indication that there is no correct barrier formation in the RhE model.

6. At the end of the reconstitution process, the tissues can be exposed to various stressors to induce for example oxidative stress or inflammation. In parallel, they can be treated with chemical compounds or cosmetic ingredients.NOTE: During the exposure/ treatment, tissues are usually maintained in submerged medium starting from ALI D14. When tissues are expected to be exposed/treated for a long period of time (i.e., 48-72 hours), it is recommended (i) to start the exposure/treatment earlier in the ALI cultivation process, such as D7-D9, to avoid the thinning of the viable layers and the thickening of the $\mathrm{SC}$, and (ii) to incubate the tissues in ALI medium, to keep stimulating cell proliferation.

7. For RhE harvesting, collect the tissues and cell culture medium at the timepoint of interest for histological 
analysis, viability assays, protein/RNA extraction, and enzyme-linked immunosorbent assays (ELISAs).

\section{Representative Results}

NHEKs cultured in 2D display a traditional morphology with a consistent polygonal shape (Figure 2A). As described above, NHEKs are seeded into culture inserts after reaching a confluency of approximately $80 \%$. The morphology of the RhEs was analyzed using H\&E staining and TEM. After 15 days at $A L I$, a fully stratified tissue is obtained as indicated by its four main epidermal layers: the SB, the SS, the SG, and the SC (Figure 2B). In the SB layer, the cells have a columnar shape. From the second layer on towards the upper layers of the RhE, NHEKs differentiate as observed by the changes in the cell morphology (from a columnar shape in the SB layer, towards a spinous shape in the SS layer). In the SG layer, the cells have a more flattened shape and display keratohyalin granules (KG) that are represented as purple dots in the cytoplasm. Their characteristic round and stellar shape is highlighted by white arrows on the H\&E image (Figure 2C). The cells in the SC, are terminally differentiated and are completely flattened and lack a cell nucleus. The stratified RhEs have an overall thickness of $84.3 \pm 2.4 \mu \mathrm{m}$ and their SC has a thickness of $19.6 \pm 3.2 \mu \mathrm{m}$ (Figure 2D). These values are comparable to those reported for native human skin, i.e., 60-120 $\mu \mathrm{m}$ and 10-20 $\mu \mathrm{m}$, respectively ${ }^{69}$. The number of viable layers is 6-7, which is lower compared to that of native human skin, being approximately $7-14^{70}$. Ultrastructural analysis of RhEs at different time points in the reconstitution protocol (i.e., $7,10,13$, and 15 days) reveals the cornification process of the RhEs with an increased number of corneocyte layers over time (Figure 2E). After 15 days at the ALI, the SC of the RhE tissue is made of approximately 15-25 layers, which is comparable to the value reported for native human skin (i.e., 15-20 layers) ${ }^{69}$. 

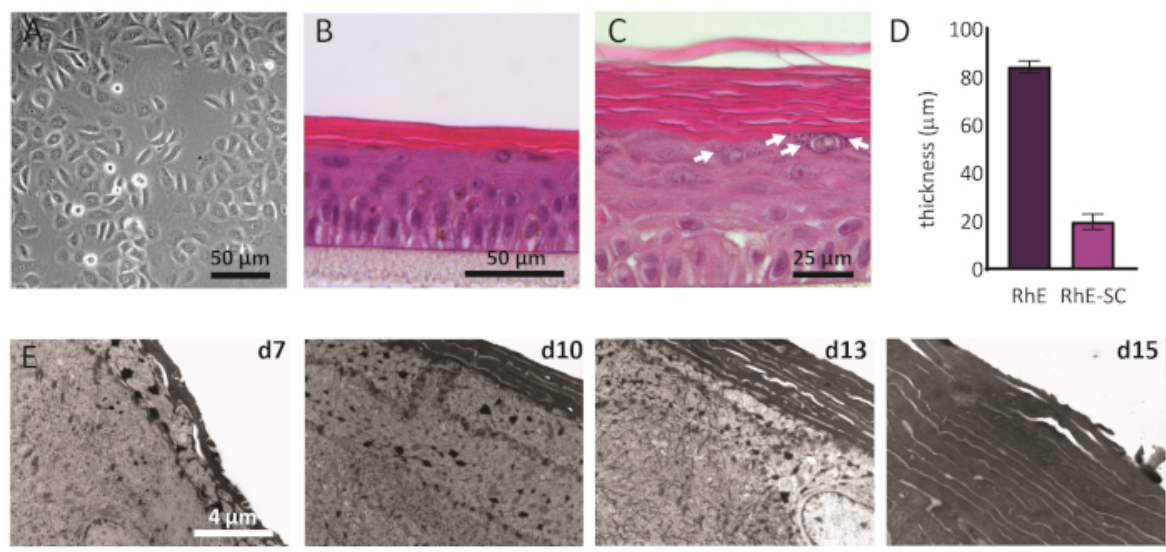

Figure 2: Primary keratinocytes and reconstructed human epidermis. (A) Phase-contrast microscopy image of primary keratinocytes before seeding onto inserts. Scale bar is $50 \mu \mathrm{m}$. (B-C) H\&E bright field microscopy image of RhE. Scale bar is $50 \mu \mathrm{m}(\mathrm{B})$ and $25 \mu \mathrm{m}$ (C). (D) Quantification of thickness of RhE and SC (mean \pm SEM, $n=3$ ). (E) Transmission electron microscopy images of RhE cross-sections after 7, 10, 13, and 15 days at ALI. Scale bar is $4 \mu \mathrm{m}$. Please click here to view a larger version of this figure.

According to their differentiation stage, NHEKs growing in 3D show different protein expression profiles according to their differentiation stage. The expression of proteins specific for early-stage keratinocyte differentiation (i.e., keratin 10), latestage keratinocyte differentiation (i.e., involucrin, loricrin, and filaggrin), and keratinocyte adhesion (i.e., desmoglein 1) in RhEs was determined using IF staining. Involucrin expression appears more predominantly located in the SG layer since its expression is initiated earlier during the differentiation process (Figure 3D), whereas filaggrin and loricrin are expressed in the upper layers (Figure 3B-C). Keratin 10 expression was found in all the viable layers, except of the SB layer (Figure 3E). RhEs display functional desmosomal junctions, as indicated by the expression of desmoglein 1 in the intercellular space of the viable epidermal layers (Figure 3F). To conclude, all five markers are expressed and located in the appropriate epidermal layers and translate to a healthy epidermal differentiation process. 

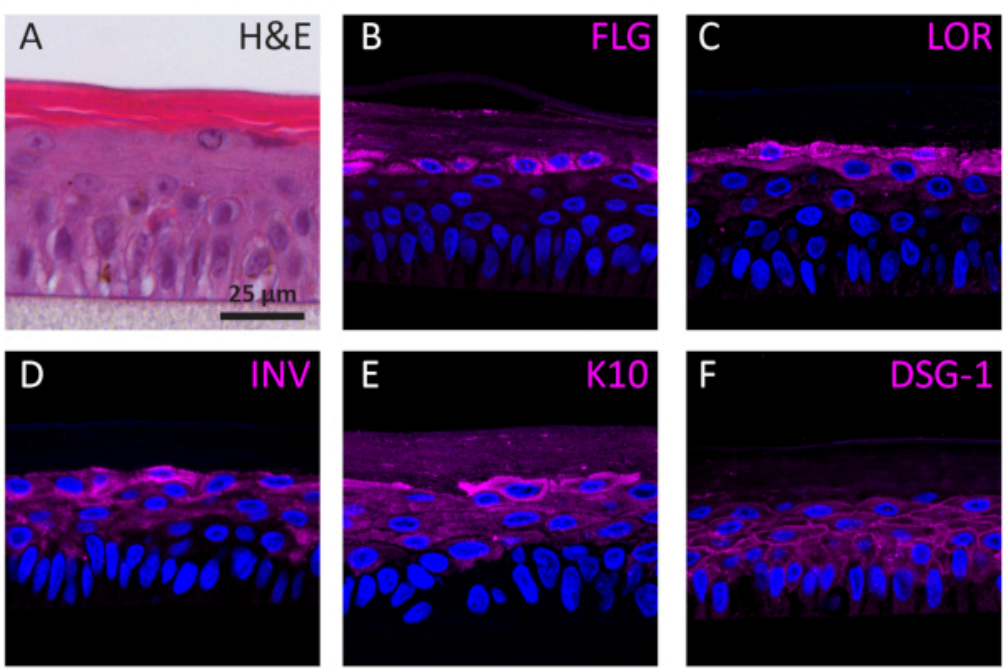

Figure 3: Epidermal differentiation, tissue adhesion, and tissue integrity of reconstructed human epidermis. (A) H\&E bright field microscopy image of RhE. Confocal fluorescence microscopy images of (B) filaggrin (FLG), (C) loricrin (LOR), (D) involucrin (INV), (E) keratin 10 (K10), and (F) desmoglein 1 (DSG-1) represented in magenta. Nuclei staining (DAPI) is represented in blue. Scale bar is $25 \mu \mathrm{m}$. Please click here to view a larger version of this figure.

The barrier properties of the RhE model was investigated by assessing both the tissue viability and integrity. The tissue integrity was determined after 15 days by measuring the TEER using a voltohmmeter. The $2567 \pm 415 \Omega . \mathrm{cm}^{2}$ values recorded for the RhEs translate the formation of a continuous barrier (Figure 4A). Those values are in range with those reported for RhE models ${ }^{71,72,73,74}$. Additionally, the required exposure time for a cytotoxic reference chemical (i.e., Triton X-100) to reduce the tissue viability by $50 \%$ (ET50) was determined with a thiazolyl blue tetrazolium bromide (MTT) assay. The ET50 value measured for the RhE was 2.1 hours. This value falls within the acceptance range of other 3D epidermal models that are qualified for reliable prediction of irritation classification (OECD Guideline 439) ${ }^{19}$. 

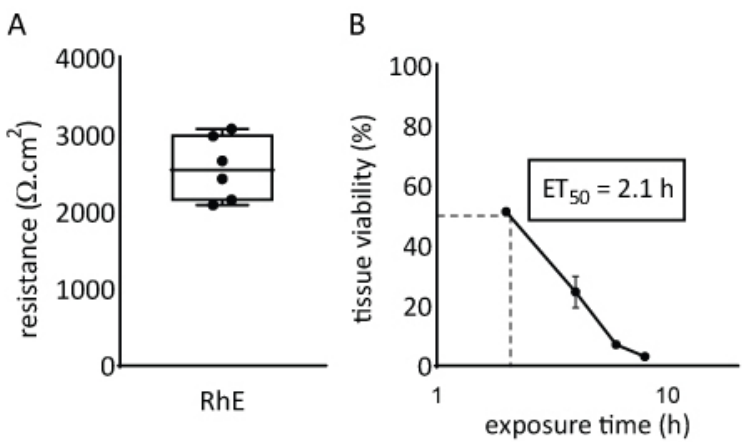

Figure 4: Barrier properties of reconstructed human epidermis. (A) Tissue integrity measured with transepithelial electrical resistance (mean \pm SEM, n=6). (B) ET50 determined by measuring tissue viability (i.e., MTT assay) upon topical exposure to $78.3 \mu \mathrm{L}$ of $1 \%$ Triton $\mathrm{X}-100$ (mean \pm SEM, $n=3$ ).

Responsiveness of RhEs was investigated upon known proinflammatory stimuli. RhEs were treated systemically, i.e., addition of stimuli in the medium of the basolateral compartment, using $100 \mu \mathrm{g} / \mathrm{mL}$ Escherichia coli lipopolysaccharide (LPS) and $40 \mathrm{ng} / \mathrm{mL}$ tumor necrosis factor alpha (TNF- $\alpha)$. After 24 hours of stimuli, the cell culture medium was collected. The cytotoxicity was measured using a lactate dehydrogenase (LDH) assay and compared to values of a known membrane disruptor, the Triton X-100 detergent (Figure 5). A significant increase $(p<0.05$, oneway ANOVA, Dunnett's multiple comparison test) was shown in LDH activity in RhEs treated with Triton X-100. LPS and TNF- $\alpha$ treatments both showed not be cytotoxic. 


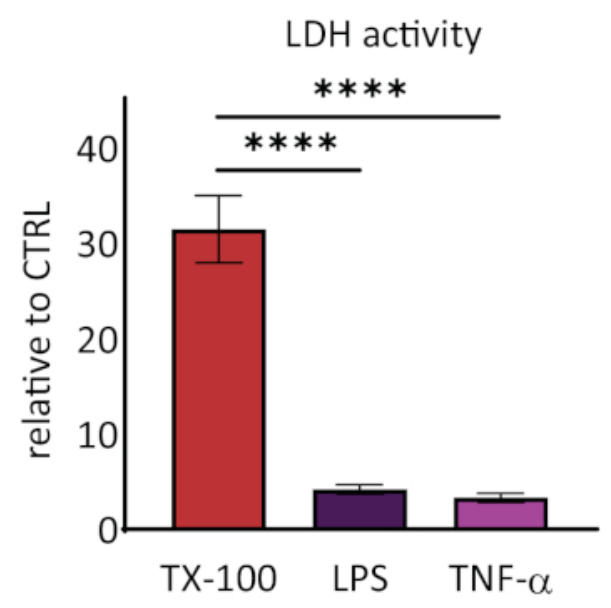

Figure 5: The cytotoxicity measured via lactate dehydrogenase (LDH) assay. Data are presented as relative values to control, untreated tissues (CTRL); mean \pm SEM, $n=9$ (Triton X-100), $n=8$ (LPS), $n=3$ (TNF- $\alpha$ ). Significance was tested with one-way ANOVA, Dunnett's multiple comparison test. Asterisk denotes statistically significant difference compared to CTRL, $\left.{ }^{* * *} p<0.0001\right)$. Please click here to view a larger version of this figure.

The release of interleukin 1 alpha (IL-1 $\alpha)$ and interleukin 8 (IL-8) in the RhE medium was quantified using ELISAs. Figure 6 shows both the quantified and relative IL-1 $\alpha$ and IL-8 release by the RhEs upon challenge with LPS and TNF- $\alpha$. LPS treatment resulted in a statistically significant $(p<0.05$, unpaired Student's T-test) induced release of IL-8 (9.6 $\pm \quad$ increase).
1.0 fold increase) and IL-1 $\alpha(2.7 \pm 1.3$ fold increase $)$. TNFa did not significantly induce IL-8 release, even though a tendency of increased IL-8 levels was observed $(2.3 \pm 0.8$ fold increase). However, TNF- $\alpha$ did significantly $(p<0.05$, unpaired Student's T-test) trigger IL-1 $\alpha$ release $(1.8 \pm 0.5$ fold 
A

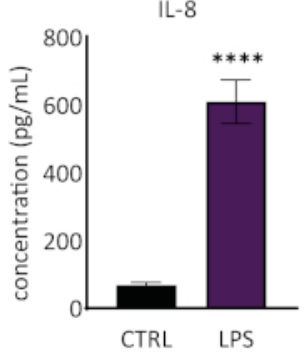

D

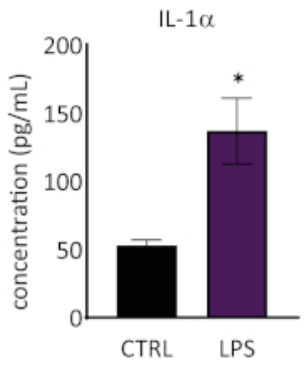

B

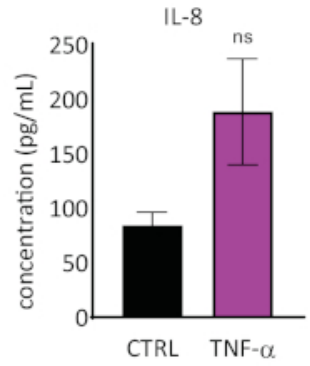

$\mathrm{E}$

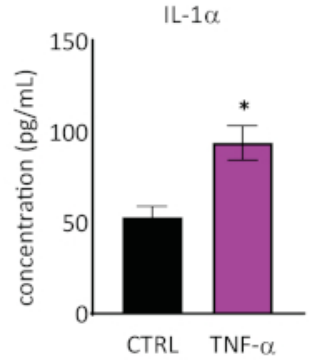

C

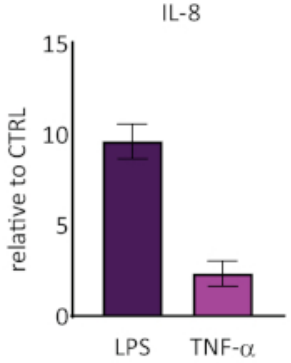

$\mathrm{F}$

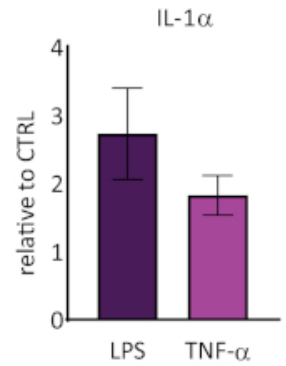

Figure 6: Proinflammatory responses in the reconstructed human epidermis. Concentrations of IL-8 release by the RhE upon a 24-hour challenge with LPS $(\mathbf{A})$ and TNF- $\alpha$ (B). Data is represented as mean \pm SEM, $n=8$ (LPS), $n=3$ (TNFa). (C) Data is represented as the mean of relative value compared to control, untreated tissues (CTRL) $\pm S E M, n=8$ (LPS), $n=3(T N F-\alpha)$. The concentration of IL-1 $\alpha$ release of the RhE upon a 24-hour challenge with LPS (D) and TNF- $\alpha(E)$. Data is represented as mean \pm SEM, $n=8$ (LPS), $n=3$ (TNF- $\alpha$ ). ( $F$ ) Data is represented as the mean of relative value compared to $\mathrm{CTRL} \pm \mathrm{SEM}, \mathrm{n}=4$ (LPS), $\mathrm{n}=3$ (TNF- $\alpha$ ). Significance was tested by an unpaired Student's T-test. Asterisk denotes statistically significant differences compared to CTRL, $\left.{ }^{*} p<0.05,{ }^{* * *} p<0.0001\right)$. Please click here to view a larger version of this figure.

\section{Discussion}

RhEs are widely used as screening tools in the pharmaceutical and dermato-cosmetic fields ${ }^{36,75,76,77,78}$. Although several companies have made such RhEs commercially available, they remain costly and limit the possibility to vary cultivation parameters as required to address new research questions. This paper describes the production procedure of in-house RhEs in a robust and reliable manner and provides a detailed characterization of the obtained tissues to confirm the relevance of the model as an alternative approach to animal testing.
Some of the steps in the protocol are crucial to assure proper keratinocyte differentiation and RhE reproducibility. This can be carried out by utilizing the optimal cells, medium type(s), and cultivation conditions. In the proposed RhE model, neonatal NHEKs were selected for their lack of antigenic exposure, compared to adult NHEKs. Furthermore, keratinocytes were limited to Caucasian ethnicity to avoid inter-species variability. Primary keratinocytes are typically used for their ability to differentiate and stratify ${ }^{79}$. They can be obtained commercially or by in-house isolation from adult $\operatorname{skin}^{80}$. The cultivation of a cell line (i.e., HaCaT) on a polycarbonate membrane, has shown to fail differentiation 
and demonstrated an impaired capacity to synthesize lipids that are necessary for barrier formation ${ }^{81}$. However, the inclusion of different culture matrices, such as hydrogels, collagen, fibrin, and spheroids cultures, resulted in the successful development of 3D skin models $78,82,83,84,85,86$ The immortalized cell lines, N/TERT, have been shown to be suitable for the development of RhEs ${ }^{35}$. Primary keratinocytes remain proliferative upon their fourth or fifth passage ${ }^{61}$, therefore the current protocol includes the use of keratinocytes in their third passage. De Vuyst et al. have demonstrated that the cell seeding density is of importance and needs to be sufficient (i.e., $\geq 2.5 \times 10^{5}$ cells $/ \mathrm{cm}^{2}$ ) to ensure that the medium from the basolateral compartment does not diffuse to the apical compartment. An insufficient seeding density (i.e., $<2.5 \times 10^{5}$ cells $/ \mathrm{cm}^{2}$ ) can result in an inability to form a proper barrier, which is indicated by the diffusion of medium from the basolateral to the apical compartment, resulting in submerged instead of ALI culture conditions ${ }^{62}$. Serum-free keratinocyte growth medium (see Table of Materials) was preferred for reproducibility purposes, since it offers the advantage of working with a chemically defined medium and reduces the risk of contamination. This medium has a lower calcium concentration (i.e., $60 \mu \mathrm{M}$ ) and therefore stimulates the proliferation of keratinocytes ${ }^{87}$. Increasing the calcium concentration (i.e., $1.5 \mathrm{mM}$ ) from the first step of the RhE cultivation, favors keratinocyte differentiation and skin barrier formation and homeostasis ${ }^{49}$. In addition, the ALI medium is supplemented with ascorbic acid, which has shown to be crucial for the formation of SC lipids and to promote differentiation ${ }^{52,53,54}$. The ALI medium also contains KGF, which is a growth factor secreted by fibroblasts that can bind to keratinocyte transmembrane receptors and upon activation has a dual role in differentiation and wound repair ${ }^{55,88}$. It is important to refresh the ALI medium at specific time intervals, to provide a constant supply of fresh nutrients to the RhEs.
The use of a carrier plate system is crucial for RhE cultivation at a larger scale (i.e., 24 inserts/plate). It offers the advantage of saving time, reducing the risk of contamination, and leaves less room for the introduction of human errors. It also provides the possibility to culture RhEs in a high volume of media (i.e., $1.5 \mathrm{~mL}$ ), which reduces the required number of $A L I$ medium refreshes. Additionally, it offers the possibility to transfer a full plate of inserts to a plate with fresh medium, avoiding contact with the inserts individually or uncovering the plate lid.

There are several limitations of the RhE model that should be noted. In native human skin there is an equilibrium between the proliferation of keratinocytes in the basal layer and the detachment of corneocytes in the SC (i.e., desquamation $)^{89}$. However, in vitro, desquamation does not take place. Therefore, the corneocytes remain attached to the RhE and form a thick SC that is less physiologically relevant. Hence, there is a limited cultivation timespan of RhEs. Moreover, this RhE model is simple and straightforward, since it consists of a singular cell type, i.e., the keratinocyte, which is the most abundant cell type of the epidermis. However, there are other cell types resident in the epidermis, such as melanocytes, dendritic cells (i.e., Langerhans cells), T cells (e.g., $\mathrm{CD}^{+}$cells), and Merkel cells $^{13}$. To enhance the physiological relevance of the skin model, researchers have made skin models more complex by adding melanocytes ${ }^{38}$, immune cells ${ }^{39}$, or patientderived cells ${ }^{90}$. One should keep in mind that the barrier properties of human skin models are different compared to native human skin, due to notably a different SC lipid composition and a higher barrier permeability $91,92,93,94,95$. However, several studies have reported changes in barrier properties of human skin models by the cultivation under hypoxia $^{96}$ or decreased relative humidity ${ }^{97}$, the modulation of the dermal matrix with chitosan ${ }^{98}$, and alteration in 
the free fatty acids in the culture medium ${ }^{99}$. Moreover, in both simple and more complex RhEs, the culture conditions and medium composition can be modulated to mimic pathological features. By challenging the model with cytokines, an abnormal morphology 100 and alterations in gene and protein expression levels can be established that are typically observed in common skin disorders, such as atopic dermatitis and psoriasis $35,101,102,103,104,105$ Silencing specific genes in keratinocytes before initiating the reconstitution process of the $3 \mathrm{D}$ model is another approach used to mimic features of skin disorders and investigate new therapeutic solutions ${ }^{106,107}$. Besides modeling an epidermal layer only, a dermal compartment can be included in the model (i.e., named human skin equivalents or full-thickness models) by embedding fibroblasts in a collagen matrix prior to RhE reconstitution, making it more physiologically relevant and suitable for aging and wound healing related studies $^{108,109,110}$. Additionally, tumor spheroids have been added to human skin equivalents to study melanoma progression ${ }^{111}, 112$. The latest advances in the field of skin models are bio-printing and skin-on-a-chip. Multiple research groups have succeeded recently in the development of (perfusable) bio-printed skin equivalents $45,113,114$. The proposed protocol takes advantage of a 24-well format and a carrier plate, avoiding inserts to be handled individually. However, the study scale is still quite limited and lacks automation. By implementing the use of bio-printing or skinon-a-chip, smaller skin models can be used with more automated processes and on a larger scale.

The RhE described in this protocol has multiple similarities to the already developed and well characterized commercial epidermal models. The morphological analysis has demonstrated that although the number of viable layers in the proposed RhE model is lower compared to that of native human skin (i.e., 6-7 compared to 7-14), it is comparable to that of the EpiDerm RhE model (i.e, 8-12) ${ }^{70}$. Similarly to the EpiDerm, EpiSkin, and SkinEthic models, the upper RhE layer shows a basket-weave pattern of densely packed corneocyte layers $^{28}$. Moreover, TEM analysis revealed that the number of SC layers in the proposed RhE model (i.e., 15-25) is comparable to that of EpiDerm (i.e., 16-25) ${ }^{70}$. Overall, the proposed RhE model demonstrates a similar structure to that of other commercialized epidermal models, mimicking the native human epidermis. The tissue integrity as measured by TEER is in range with commercial RhE models (i.e., between 3000-5600 $\left.\Omega \cdot \mathrm{m}^{2}\right)^{71,72,73}$ and other in-house RhEs (i.e., approximately $\left.5000 \Omega . \mathrm{cm}^{2}\right)^{74,115}$. The proposed RhE model shows to be well differentiated, as indicated by the presence and correct localization of differentiation and tissue adhesion markers. Moreover, the proposed RhE model shows to be responsive to proinflammatory stimuli (i.e., LPS and TNF- $\alpha$ ).

To conclude, the current protocol shows how to produce RhEs in a reliable manner and at a relatively large scale to meet the needs of researchers in both academic and private institutions. The proposed RhE model shows to have similar morphology, epidermal differentiation, and biological responsiveness to other existing commercial models. It provides an alternative tool for both the pharmaceutical and dermato-cosmetic field when access to a relevant skin model is required.

\section{Disclosures}

Marc Eeman and Benedetta Petracca are employees of Dow Silicones Belgium. All other authors have nothing to disclose.

\section{Acknowledgments}

The European Union's Horizon 2020 Research and Innovation Programme under the Marie Skłodowska-Curie 
Grant with the grant agreement no. 765602 funded this work. All authors recognize the support of the Adolphe Merkle Foundation and the University of Ferrara and gratefully acknowledge Dow Silicones Belgium. Dr. Miguel SpuchCalvar is acknowledged for preparing the graphical images of the RhE cultivation process. The authors thank Dr. Barbara Drasler, Dr. Franco Cervellati, and Dr. Agnès Tessier for their technical support and discussions.

\section{References}

1. Alberts, B., Johnson, A., Lewis, J. Molecular Biology of the Cell. 4th edition. Antioxidants \& Redox Signaling. (2002).

2. Pouillot, A., Dayan, N., Polla, A.S., Polla, L.L., Polla, B.S. The stratum corneum: a double paradox. Journal of Cosmetic Dermatology. 7 (2), 143-148 (2008).

3. Moore, K.L., Dalley, A.F., A.A. Clinically Orientated Anatomy. (2010).

4. Barthel, R., Aberdam, D. Epidermal stem cells. Journal of the European Academy of Dermatology and Venereology. 19 (4), 405-413 (2005).

5. Green, K.J., Simpson, C.L. Desmosomes: new perspectives on a classic. Journal of Investigative Dermatology. 127 (11), 2499-2515 (2007).

6. Feingold, K.R. Lamellar bodies: the key to cutaneous barrier function. Journal of Investigative Dermatology. 132 (8), 1951-1953 (2012).

7. Elias, M.P., Feingold, K.R., Fartasch, M. The epidermal lamellar body as a multifunctional secretory organelle. Skin Barrier. 261-72 (2006).

8. Tobin, D.J. Biochemistry of human skin-our brain on the outside. Chemical society reviews. 35 (1), 52-67 (2006).
9. Bouwstra, J.A., Ponec, M. The skin barrier in healthy and diseased state. Biochimica et Biophysica Acta (BBA) Biomembranes. 1758 (12), 2080-2095 (2006).

10. Weinstein, G.D., McCullough, J.L., Ross, P. Cell proliferation in normal epidermis. Journal of Investigative Dermatology. 82 (6), 623-628 (1984).

11. Madison, K.C. Barrier function of the skin: "la raison d'être" of the epidermis. Journal of Investigative Dermatology. 121 (2), 231-241 (2003).

12. Suter, M.M. et al. The keratinocyte in epidermal renewal and defence. Veterinary Dermatology. 20 (5-6), 515-532 (2009).

13. Ella McLafferty, Charles Hendry, F.A. The integumentary system: anatomy, physiology and function of skin. Nursing Standard. 27 (7), 35-43 (2012).

14. Monteiro-Riviere, N.A. Toxicology of the skin. (2010).

15. Dellambra, E., Odorisio, T., D'Arcangelo, D., Failla, C.M., Facchiano, A. Non-animal models in dermatological research. ALTEX. 36 (2), 177-202 (2019).

16. Gordon, S. et al. Non-animal models of epithelial barriers (skin, intestine and lung) in research, industrial applications and regulatory toxicology. Altex. 32 (4), 327-378 (2015).

17. Kandárová, $H$. et al. The EpiDerm test protocol for the upcoming ECVAM validation study on in vitro skin irritation tests - An assessment of the performance of the optimised test. Alternatives to laboratory animals : ATLA. 33 (4), 351-367 (2005).

18. Kandárová, $H$. et al. Assessment of the skin irritation potential of chemicals by using the SkinEthic reconstructed human epidermal model and the common skin irritation protocol evaluated in the ECVAM skin 
irritation validation study. Alternatives to laboratory animals : ATLA. 34 (4), 393-406 (2006).

19. Test No. 439: In Vitro Skin Irritation: Reconstructed Human Epidermis Test Method. OECD. (2019).

20. Alépée, N., Grandidier, M.H., Cotovio, J. Subcategorisation of skin corrosive chemicals by the EpiSkin $^{\mathrm{TM}}$ reconstructed human epidermis skin corrosion test method according to UN GHS: Revision of OECD Test Guideline 431. Toxicology in Vitro. 28 (2), 131-145 (2014).

21. Test No. 431: In vitro skin corrosion: reconstructed human epidermis (RHE) test method. OECD. (2019).

22. Mehling, A. et al. In vitro RHE skin sensitisation assays: applicability to challenging substances. Regulatory Toxicology and Pharmacology. 108, 104473 (2019).

23. SENS-IS | EURL ECVAM - TSAR. at <https:// tsar.jrc.ec.europa.eu/test-method/tm2011-11>. (2011).

24. Lelièvre, D. et al. The episkin phototoxicity assay (EPA): development of an in vitro tiered strategy using 17 reference chemicals to predict phototoxic potency. Toxicology in Vitro. 21 (6), 977-995 (2007).

25. Flaten, G.E. et al. In vitro skin models as a tool in optimization of drug formulation. European Journal of Pharmaceutical Sciences. 75, 10-24 (2015).

26. Pellevoisin, C., Bouez, C., Cotovio, J. Cosmetic industry requirements regarding skin models for cosmetic testing. Skin Tissue Models. 3-37 (2018).

27. Niehues, $H$. et al. 3D skin models for $3 R$ research: the potential of $3 \mathrm{D}$ reconstructed skin models to study skin barrier function. Experimental Dermatology. 27 (5), 501-511 (2018).
28. Netzlaff, F., Lehr, C.-M., Wertz, P.W., Schaefer, U.F. The human epidermis models EpiSkin $₫$, SkinEthic $\circledast$ and EpiDerm $®$ : An evaluation of morphology and their suitability for testing phototoxicity, irritancy, corrosivity, and substance transport. European Journal of Pharmaceutics and Biopharmaceutics. 60 (2), 167-178 (2005).

29. Prieux, R., Eeman, M., Rothen-Rutishauser, B., Valacchi, G. Mimicking cigarette smoke exposure to assess cutaneous toxicity. Toxicology in Vitro. 62, 104664 (2020).

30. Petracca, B., Rothen-Rutishauser, B., Valacchi, G., Eeman, M. Bench approaches to study the detrimental cutaneous impact of troposperic ozone. Journal of Exposure Science and Environmental Epidemiology. 37, 137-148 (2021).

31. Dijkhoff, I.M. et al. Impact of airborne particulate matter on skin: a systematic review from epidemiology to in vitro studies. Particle and fibre toxicology. 17 (1), 35 (2020).

32. El Ghalbzouri, A., Siamari, R., Willemze, R., Ponec, M. Leiden reconstructed human epidermal model as a tool for the evaluation of the skin corrosion and irritation potential according to the ECVAM guidelines. Toxicology in Vitro. 22 (5), 1311-1320 (2008).

33. Chacón, M. et al. Development of an in-house reconstructed human epidermis model as an alternative method in skin corrosion assessment. Toxicology in Vitro. 65, 104779 (2020).

34. Pedrosa, T. do N. et al. A new reconstructed human epidermis for in vitro skin irritation testing. Toxicology in Vitro. 42, 31-37 (2017). 
35. Smits, J.P.H. et al. Immortalized N/TERT keratinocytes as an alternative cell source in $3 \mathrm{D}$ human epidermal models. Scientific Reports. 7 (1), 11838 (2017).

36. Poumay, Y., Coquette, A. Modelling the human epidermis in vitro: tools for basic and applied research. Archives of dermatological research. 298 (8), 361-9 (2007).

37. Rikken, G., Niehues, H., van den Bogaard, E.H. Organotypic 3D skin models: human epidermal equivalent cultures from primary keratinocytes and immortalized keratinocyte cell lines. Methods in Molecular Biology. 2154, 45-61 (2020).

38. Duval, C. et al. Human skin model containing melanocytes: essential role of keratinocyte growth factor for constitutive pigmentation-functional response to $\alpha$ melanocyte stimulating hormone and forskolin. Tissue engineering. Part C, Methods. 18 (12), 947-57 (2012).

39. Hutter, V., Kirton, S.B., Chau, D.Y.S. Immunocompetent human in vitro skin models. Skin Tissue Models. 353-373 (2018).

40. Kinsner, A., Lesiak-Cyganowska, E., Śladowski, D. In vitro reconstruction of full thickness human skin on a composite collagen material. Cell and Tissue Banking. 2 (3), 165-171 (2001).

41. Black, A.F., Bouez, C., Perrier, E., Schlotmann, K., Chapuis, F., Damour, O. Optimization and characterization of an engineered human skin equivalent. Tissue Engineering. 11 (5-6), 723-733 (2005).

42. Reijnders, C.M.A. et al. Development of a full-thickness human skin equivalent in vitro model derived from
TERT-immortalized keratinocytes and fibroblasts. Tissue Engineering. Part A. 21 (17-18), 2448-2459 (2015).

43. Groeber, F., Holeiter, M., Hampel, M., Hinderer, S., Schenke-Layland, K. Skin tissue engineering - In vivo and in vitro applications. Advanced Drug Delivery Reviews. 63 (4-5), 352-366 (2011).

44. Mathes, S.H., Ruffner, H., Graf-Hausner, U. The use of skin models in drug development. Advanced Drug Delivery Reviews. 69-70, 81-102 (2014).

45. Kim, B.S., Gao, G., Kim, J.Y., Cho, D. 3D cell printing of perfusable vascularized human skin equivalent composed of epidermis, dermis, and hypodermis for better structural recapitulation of native skin. Advanced Healthcare Materials. 8 (7), e1801019 (2019).

46. Pittelkow, M.R., Scott, R.E. New techniques for the in vitro culture of human skin keratinocytes and perspectives on their use for grafting of patients with extensive burns. Mayo Clinic Proceedings. 61 (10), 771-777 (1986).

47. Elias, P.M., Ahn, S.K., Brown, B.E., Crumrine, D., Feingold, K.R. Origin of the epidermal calcium gradient: regulation by barrier status and role of active vs passive mechanisms. Journal of Investigative Dermatology. 119 (6), 1269-1274 (2002).

48. Elias, P.M. et al. Modulations in epidermal calcium regulate the expression of differentiation-specific markers. Journal of Investigative Dermatology. 119 (5), 1128-1136 (2002).

49. Lee, S.E., Lee, S.H. Skin barrier and calcium. Annals of Dermatology. 30 (3), 265-275 (2018).

50. Prunieras, M., Regnier, M., Woodley, D. Methods for cultivation of keratinocytes with an air-liquid interface. 
Journal of Investigative Dermatology. 81 (1 Suppl), 28s-33s (1983).

51. Poumay, Y. et al. A simple reconstructed human epidermis: preparation of the culture model and utilization in in vitro studies. Archives of Dermatological Research. 296 (5), 203-211 (2004).

52. Ponec, M. et al. The formation of competent barrier lipids in reconstructed human epidermis requires the presence of vitamin C. Journal of Investigative Dermatology. 109 (3), 348-355 (1997).

53. Savini, I. et al. Characterization of keratinocyte differentiation induced by ascorbic acid: Protein kinase C involvement and vitamin C homeostasis. Journal of Investigative Dermatology. 118 (2), 372-379 (2002).

54. Pasonen-Seppänen, S. et al. Vitamin C enhances differentiation of a continuous keratinocyte cell line (REK) into epidermis with normal stratum corneum ultrastructure and functional permeability barrier. Histochemistry and Cell Biology. 116 (4), 287-297 (2001).

55. Beer, H.D. et al. Expression and function of keratinocyte growth factor and activin in skin morphogenesis and cutaneous wound repair. Journal of Investigative Dermatology Symposium Proceedings. 5 (1), 34-39 (2000).

56. Steven, A.C., Bisher, M.E., Roop, D.R., Steinert, P.M. Biosynthetic pathways of filaggrin and loricrin--two major proteins expressed by terminally differentiated epidermal keratinocytes. Journal of structural biology. 104 (1-3), 150-162 (1990).

57. Rice, R.H., Thacher, S.M. Involucrin: a constituent of cross-linked envelopes and marker of squamous maturation. Biology of the Integument. 752-761 (1986).
58. Elias, P.M., Feingold, K.R. Skin Barrier. (2011).

59. Marionnet, C. et al. Morphogenesis of dermal-epidermal junction in a model of reconstructed skin: beneficial effects of vitamin C. Experimental Dermatology. 15 (8), 625-633 (2006).

60. Frikke-Schmidt, H., Lykkesfeldt, J. Keeping the intracellular vitamin $\mathrm{C}$ at a physiologically relevant level in endothelial cell culture. Analytical Biochemistry. 397 (1), 135-137 (2010).

61. Castro-Muñozledo, F., Hernández-Quintero, M., MarschMoreno, M., Kuri-Harcuch, W. Cultivation, serial transfer, and differentiation of epidermal keratinocytes in serumfree medium. Biochemical and Biophysical Research Communications. 236 (1), 167-172 (1997).

62. De Vuyst, E. et al. Reconstruction of normal and pathological human epidermis on polycarbonate filter. Epidermal Cells. Methods in Molecular Biology (Methods and Protocols). 191-201 (2013).

63. Chen, R.H., Zhu, J., Zhang, R.Z., Wang, S.Y., Li, Y. The tolerance of human epidermal cells to trypsinization in vitro. Cell and Tissue Banking. 21 (2), 257-264 (2020).

64. Pohanish, R.P. Sittig's handbook of toxic and hazardous chemicals and carcinogens. Sittig's Handbook of Toxic and Hazardous Chemicals and Carcinogens. 2 (2012).

65. Tsaousis, K.T. et al. Time-dependent morphological alterations and viability of cultured human trabecular cells after exposure to Trypan blue. Clinical and Experimental Ophthalmology. 41 (5), 484-490 (2013).

66. Kim, S.I. et al. Application of a non-hazardous vital dye for cell counting with automated cell counters. Analytical Biochemistry. 492, 8-12 (2016). 
67. Lundholt, B.K., Scudder, K.M., Pagliaro, L. A simple technique for reducing edge effect in cell-based assays. Journal of Biomolecular Screening. 8 (5), 566-570 (2003).

68. Fartasch, M., Ponec, M. Improved barrier structure formation in air-exposed human keratinocyte culture systems. Journal of Investigative Dermatology. 102 (3), 366-374 (1994).

69. Bouwstra, J.A., Honeywell-Nguyen, P.L., Gooris, G.S., Ponec, M. Structure of the skin barrier and its modulation by vesicular formulations. Progress in Lipid Research. $\mathbf{4 2}$ (1), 1-36 (2003).

70. Ponec, M., Boelsma, E., Gibbs, S., Mommaas, M. Characterization of reconstructed skin models. Skin Pharmacology and Physiology. 15 (1), 4-17 (2002).

71. Hubaux, R., Wauters, A., Chrétien, A., Poumay, Y., Salmon, M. Reconstructed human epidermis response to urban particulate matter activates multiple stress-related pathways and impacts the skin barrier function. 23th IFSCC Conference. 125-134 (2017).

72. Lin, Y.-C. et al. Testing method development and validation for in vitro skin irritation testing (SIT) by using reconstructed human epidermis (RhE) skin equivalent EPiTRI®. Alternatives to Animal Testing. 8-19 (2019).

73. Alexander, F.A., Eggert, S., Wiest, J. Skin-on-a-chip: Transepithelial electrical resistance and extracellular acidification measurements through an automated airliquid interface. Genes. 9 (2), 114 (2018).

74. van den Bogaard, E. et al. Perspective and consensus opinion: good practices for using organotypic skin and epidermal equivalents in experimental dermatology research. Journal of Investigative Dermatology. 141, (1) 203-205 (2019).

75. Kandárová, H., Hayden, P., Klausner, M., Kubilus, J., Sheasgreen, J. An in vitro skin irritation test (SIT) using the EpiDerm reconstructed human epidermal (RHE) model. Journal of Visualized Experiments. (29), 1366 (2009).

76. Abd, E. et al. Skin models for the testing of transdermal drugs. Clinical pharmacology: advances and applications. 8, 163-176 (2016).

77. De Wever, B., Kurdykowski, S., Descargues, P. Human skin models for research applications in pharmacology and toxicology: introducing nativeSkin, the "missing link" bridging cell culture and/or reconstructed skin models and human clinical testing. Applied In Vitro Toxicology. 1 (1), 26-32 (2015).

78. Klicks, J., von Molitor, E., Ertongur-Fauth, T., Rudolf, R., Hafner, M. In vitro skin three-dimensional models and their applications. Journal of Cellular Biotechnology. 3 (1), 21-39 (2017).

79. Bernstam, L.I., Vaughan, F.L., Bernstein, I.A. Keratinocytes grown at the air-liquid interface. In Vitro Cellular and Developmental Biology - Animal. 22 (12), 695-705 (1986).

80. Johansen, C. Generation and culturing of primary human keratinocytes from adult skin. Journal of Visualized Experiments. (130), 56863 (2017).

81. Boelsma, E., Verhoeven, M.C.H., Ponec, M. Reconstruction of a human skin equivalent using a spontaneously transformed keratinocyte cell line (HaCaT). Journal of Investigative Dermatology. 112 (4), 489-498 (1999). 
82. Zhao, X. et al. Photocrosslinkable gelatin hydrogel for epidermal tissue engineering. Advanced Healthcare Materials. 5 (1), 108-118 (2016).

83. Peura, M. et al. Paracrine factors from fibroblast aggregates in a fibrin-matrix carrier enhance keratinocyte viability and migration. Journal of Biomedical Materials Research. Part A. 95 (2), 658-664 (2010).

84. Schoop, V.M., Mirancea, N., Fusenig, N.E. Epidermal organization and differentiation of HaCat keratinocytes in organotypic coculture with human dermal fibroblasts. Journal of Investigative Dermatology. 112 (3), 343-353 (1999).

85. Lee, $\mathrm{V}$. et al. Design and fabrication of human skin by three-dimensional bioprinting. Tissue engineering. Part C, Methods. 20 (6), 473-84 (2014).

86. Alameda, J.P. et al. IKKa regulates the stratification and differentiation of the epidermis: Implications for skin cancer development. Oncotarget. 7 (47), 76779-76792 (2016).

87. Bikle, D.D., Xie, Z., Tu, C.L. Calcium regulation of keratinocyte differentiation. Expert Review of Endocrinology and Metabolism. 7 (4), 461-472 (2012).

88. Staiano-Coico, L. et al. Human keratinocyte growth factor effects in a porcine model of epidermal wound healing. Journal of Experimental Medicine. 178 (3), 865-878 (1993).

89. Egelrud, T. Desquamation in the stratum corneum. Acta Dermato-Venereologica. 80 (Supp 208), 44-45 (2000).

90. Jean, J., Lapointe, M., Soucy, J., Pouliot, R. Development of an in vitro psoriatic skin model by tissue engineering. Journal of Dermatological Science. 53 (1), 19-25 (2009).
91. Lotte, C., Patouillet, C., Zanini, M., Messager, A., Roguet, R. Permeation and skin absorption: reproducibility of various industrial reconstructed human skin models. Skin Pharmacology and Applied Skin Physiology. 15 (Suppl 1), $18-30$ (2002).

92. Ponec, M., Weerheim, A., Lankhorst, P., Wertz, P. New acylceramide in native and reconstructed epidermis. Journal of Investigative Dermatology. 120 (4), 581-588 (2003).

93. Thakoersing, V.S. et al. Unraveling barrier properties of three different in-house human skin equivalents. Tissue engineering. Part C, Methods. 18 (1), 1-11 (2012).

94. Thakoersing, V.S. et al. Increased presence of monounsaturated fatty acids in the stratum corneum of human skin equivalents. Journal of Investigative Dermatology. 133 (1), 59-67 (2013).

95. Van Smeden, J. et al. Combined LC/MS-platform for analysis of all major stratum corneum lipids, and the profiling of skin substitutes. Biochimica et Biophysica Acta - Molecular and Cell Biology of Lipids. 1841 (1), 70-79 (2014).

96. Mieremet, A. et al. Human skin equivalents cultured under hypoxia display enhanced epidermal morphogenesis and lipid barrier formation. Scientific Reports. 9 (1), 7811 (2019).

97. Mieremet, A. et al. Unravelling effects of relative humidity on lipid barrier formation in human skin equivalents. Archives of Dermatological Research. 311 (9), 679-689 (2019).

98. Mieremet, A., Rietveld, M., Absalah, S., Van Smeden, J., Bouwstra, J.A., El Ghalbzouri, A. Improved epidermal barrier formation in human skin models by Chitosan 
modulated dermal matrices. PLOS ONE. 12 (3), e0174478 (2017).

99. Mieremet, A. et al. Contribution of palmitic acid to epidermal morphogenesis and lipid barrier formation in human skin equivalents. International Journal of Molecular Sciences. 20 (23), 6069 (2019).

100.Boniface, K. et al. IL-22 inhibits epidermal fifferentiation and induces proinflammatory gene expression and migration of human keratinocytes. The Journal of Immunology. 174 (6), 3695-3702 (2005).

101.De Vuyst, E., Salmon, M., Evrard, C., Lambert de Rouvroit, C., Poumay, Y. Atopic dermatitis studies through in vitro models. Frontiers in Medicine. 4, 119 (2017).

102.Danso, M.O. et al. TNF- $\alpha$ and Th2 cytokines induce atopic dermatitis-like features on epidermal differentiation proteins and stratum corneum lipids in human skin equivalents. Journal of Investigative Dermatology. 134 (7), 1941-1950 (2014).

103.Soboleva, A.G., Mezentsev, A., Zolotorenko, A., Bruskin, S., Pirusian, E. Three-dimensional skin models of psoriasis. Cells Tissues Organs. 199 (5-6), 301-310 (2014).

104.Desmet, E., Ramadhas, A., Lambert, J., Gele, M. Van In vitro psoriasis models with focus on reconstructed skin models as promising tools in psoriasis research. Experimental Biology and Medicine. 242 (11), 1158-1169 (2017).

105.Niehues, H., van den Bogaard, E.H. Past, present and future of in vitro $3 \mathrm{D}$ reconstructed inflammatory skin models to study psoriasis. Experimental Dermatology. 27 (5), 512-519 (2018).
106. Pendaries, V. et al. Knockdown of filaggrin in a threedimensional reconstructed human epidermis impairs keratinocyte differentiation. Journal of Investigative Dermatology. 134 (12), 2938-2946 (2014).

107.Niehues, $\mathrm{H}$. et al. Epidermal equivalents of filaggrin null keratinocytes do not show impaired skin barrier function. Journal of Allergy and Clinical Immunology. 139 (6), 1979-1981.e13 (2017).

108.Reuter, C., Walles, H., Groeber, F. Preparation of a three-dimensional full thickness skin equivalent. Methods in Molecular Biology. 1612, 191-198 (2017).

109.Bataillon, M. et al. Characterization of a new reconstructed full thickness skin model, t-skin ${ }^{\mathrm{TM}}$, and its application for investigations of anti-aging compounds. International Journal of Molecular Sciences. 20 (9), 2240 (2019).

110.Rossi, A., Appelt-Menzel, A., Kurdyn, S., Walles, H., Groeber, F. Generation of a three-dimensional full thickness skin equivalent and automated wounding. Journal of Visualized Experiments. (96), 52576 (2015).

111.Li, L., Fukunaga-Kalabis, M., Herlyn, M. The threedimensional human skin reconstruct model: a tool to study normal skin and melanoma progression. Journal of Visualized Experiments. (54), 2937 (2011).

112.Müller, I., Kulms, D. A 3D organotypic melanoma spheroid skin model. Journal of Visualized Experiments. (135), 57500 (2018).

113.Wei, Z. et al. Two-dimensional cellular and threedimensional bio-printed skin models to screen topicaluse compounds for irritation potential. Frontiers in Bioengineering and Biotechnology. 8, 109 (2020). 
114.Derr, K. et al. Fully three-dimensional bioprinted skin equivalent constructs with validated morphology and barrier function. Tissue Engineering - Part C: Methods. 25 (6), 334-343 (2019).

115. Frankart, A. et al. Epidermal morphogenesis during progressive in vitro $3 \mathrm{D}$ reconstruction at the air-liquid interface. Experimental Dermatology. 21 (11), 871-875 (2012). 